\title{
REPLY TO EZCURDIA AND GÓMEZ-TORRENTE
}

\author{
ScotT SoAmes \\ School of Philosophy \\ University of Southern California \\ soames@usc.edu
}

SUMmarY: Contra Ezcurdia, it is argued that my thesis — that substitution of coreferential names or indexicals in attitude ascriptions preserves truth values of propositions semantically expressed, although it often changes truth values of propositions asserted - is compatible with the fact that belief ascriptions play important explanatory roles. Contra Gomez-Torrente, it is argued that although single-word natural kind terms are rigid in Kripke's original sense, natural kind predicates containing them are neither rigid nor obstinately essential -in the sense of applying to the same individuals in every possible world-state, whether those individuals exist at the world-state or not.

KEY WORDS: names, natural kind terms, belief ascriptions, essentialist predicates

RESUMEN: Contra Ezcurdia, se argumenta que mi tesis de que la sustitución de nombres o deícticos correferenciales en adscripciones de actitudes proposicionales preserva los valores de verdad de las proposiciones expresadas semánticamente, aunque a menudo cambia los valores de verdad de las proposiciones aseveradas, es compatible con el hecho de que las adscripciones de creencias desempeñan papeles explicativos importantes. Contra GómezTorrente, se argumenta que aunque los términos de clase natural de una sola palabra son rígidos en el sentido original de Kripke, los predicados de clase natural que los contienen no son ni rígidos ni obstinadamente esenciales, en el sentido de que se aplican a los mismos individuos en todos los mundos posibles, sea que esos individuos existan o no existan en ese mundo.

PALABRAS CLAVE: nombres, términos de clase natural, adscripciones de creencias, predicados esencialistas

Thanks to Maite Ezcurdia and Mario Gómez-Torrente for challenging and well-informed essays that advance the discussion of several topics raised in Beyond Rigidity (2002). In what follows, I will respond to their most important points. 
Reply to Gómez-Torrente

Gómez-Torrente argues for three main theses.

Tl. The notions of a weakly, a persistently, and an obstinately essential predicate correspond to the notions of a weakly, a persistently, and an obstinately rigid singular term. This correspondence consists in the fact that the two sets of notions play analogous roles in the derivations of the truth of certain statements of necessity from the truth of related identity statements involving the relevant terms. In particular, the statement of necessity the truth of which is derivable from the truth of an identification statement $\forall \boldsymbol{x}(\boldsymbol{A x} \leftrightarrow \boldsymbol{B} \boldsymbol{x})$ involving obstinately essential predicates $A$ and $B$ (and possibilist quantification) corresponds to the statement of necessity the truth of which is derivable from the truth of an identity statement $\boldsymbol{\alpha}=\boldsymbol{\beta}$ involving obstinately rigid singular terms $\alpha$ and $\beta$. The same holds for cases involving two weakly or persistently essential predicates, on the one hand, and two weakly or persistently rigid singular terms, on the other. ${ }^{1}$

T2. Since natural kind predicates that figure in the theoretical identification statements Kripke is interested in are obstinately essential, the statements of necessity the truth of which he is able to derive from the truth of $\forall \boldsymbol{x}(\boldsymbol{A x} \supset \boldsymbol{B} \boldsymbol{x})$ and $\forall \boldsymbol{x}(\boldsymbol{A} \boldsymbol{x} \leftrightarrow \boldsymbol{B} \boldsymbol{x})$, plus correct characterizations of the essentiality of the predicates, are Necessarily $\forall \boldsymbol{x}(\boldsymbol{A x} \supset \boldsymbol{B x})$ and Necessarily $\forall \boldsymbol{x}(\boldsymbol{A x} \leftrightarrow \boldsymbol{B} \boldsymbol{x})$. This analysis captures the force of standard Kripkean examples.

T3. These points justify taking a predicate or general term to be rigid iff it is essentialist, thereby extending the notion of a rigid designation to include these expressions, as well as singular terms.

\footnotetext{
${ }^{1}$ Boldface italics are used as corner quotes.
} 
I have no quarrel with $\mathrm{T} l$, for which Gómez-Torrente makes a strong case. However, I will argue that T2 and T3 are incorrect, and that something other than rigidity or essentiality is needed to explain the necessity of many true theoretical identification statements involving natural kind predicates.

My first point is that some natural kind predicates which figure in theoretical identification statements that are both necessary and a posteriori, and which seem to be intended by Kripke to be covered by his semantic analysis, are not essentialist at all, let alone obstinately so. This point is illustrated by the second example in the following passage from Naming and Necessity.

it turns out that a material object is (pure) gold if and only if the only element contained therein is that with atomic number 79. Here, the 'if and only if' can be taken to be strict (necessary). In general, science attempts, by investigating basic structural traits, to find the nature, and thus the essence (in the philosophical sense) of the kind. The case of natural phenomena is similar; such theoretical identifications as 'heat is molecular motion' are necessary, though not a priori. The type of property identity used in science seems to be associated with necessity, not with aprioricity, or analyticity: For all bodies $\boldsymbol{x}$ and $\boldsymbol{y}, \boldsymbol{x}$ is hotter than $\boldsymbol{y}$ if and only if $\boldsymbol{x}$ has a higher mean molecular kinetic energy than $\boldsymbol{y}$. Here the coextensiveness of the predicates is necessary, but not a priori. (1980, p. 138; my boldface emphasis.)

In this passage, Kripke gives two examples of necessary, a posteriori, theoretical identification sentences that have the form of universally quantified biconditionals. He also seems to suggest that the doubly quantified biconditional $(\mathrm{lb})$ is a proper analysis of the identity (la).

la. Heat is molecular motion.

b. For all bodies $x$ and $y, x$ is hotter than $y$ if and only if $x$ has a higher mean molecular kinetic energy than $y$.

In light of these and other examples, I concluded in Beyond Rigidity that Kripke's claims about theoretical identity sen- 
tences involving natural kind terms should be seen as encompassing cases in which the terms are used to form predicates, and the identity sentences are represented as universally quantified conditionals or biconditionals. The important point to emphasize here is that the predicate is hotter than is not even weakly essentialist, since one object can be hotter than another in one circumstance, without this being so in all possible circumstances in which the two exist. Nevertheless, (lb) is one of Kripke's paradigmatic examples of the necessary a posteriori.

This example is not an isolated one. On page 127, focusing on predicative uses of natural kind terms, Kripke says:

According to the conception I advocate, then, terms for natural kinds are much closer to proper names than is ordinarily supposed. The old term 'common name' is thus quite appropriate for predicates marking out species or natural kinds, such as 'cow' or 'tiger' [...]. (My boldface emphasis.)

A little later, Kripke reinforces the idea that his semantic analysis of natural kind terms applies to expressions of a variety of grammatical categories, including "terms for natural phenomena, such as 'heat', 'light', 'sound', 'lightning' and, presumably, suitably elaborated, to corresponding adjectives - 'hot', 'loud', "red"" (1980, p. 134). Example (lb), involving a form of the adjective 'hot', is an instance of this point. A similar example involving 'red' would have the form (2).

2. For all objects $x, x$ is red iff the percentages of light of different wavelengths reflected by $x$ is ....

Like (lb), (2) is an instance of the necessary a posteriori, even though the simple, natural kind predicate it involves - is redis not even weakly essentialist.

The significance of examples (1) and (2) is that they show that the necessity of some a posteriori statements of theoretical identification involving natural kind predicates cannot be explained by citing the rigidity of their predicates - when rigidity for predicates is defined as (any type of) essentiality. Since this is 
the strategy envisioned by Gómez-Torrente for other Kripkean examples — such as (3) and (4) - he must be contemplating two different sorts of explanation, one for statements of theoretical identification involving essentialist predicates (which he characterizes as rigid), and one for statements of theoretical identification involving nonessentialist predicates (which he characterizes as nonrigid).

3. Water is $\mathrm{H}_{2} \mathrm{O}-\forall x\left(x\right.$ is water iff $x$ is $\left.\mathrm{H}_{2} \mathrm{O}\right)$.

4. Lightning is an electrical discharge $-\forall x$ ( $x$ is lightning $\supset x$ is an electrical discharge).

By contrast, I argue in chapter 10 of Beyond Rigidity that a unified explanation of the necessity of (1-4) can be given using other aspects of Kripke's semantic analysis, above and beyond the notion of rigid designation (or essentiality of predicates). As far as I can tell, nothing in Gómez-Torrente's discussion threatens this positive approach.

My second point signals a disagreement between us about even those natural kind predicates that are essentialist. Whereas Gómez-Torrente sees predicates such as is (an instance of) water, is (an instance of) $\mathrm{H}_{2} \mathrm{O}$, is (an instance of) lightning, is an electrical discharge, is a cat, is a man, and is human as obstinately essential (and hence as applying to the same objects in each world-state $w$, whether or not the objects exist at $w$ ), I believe these predicates to be only persistently essential (and hence to apply at $w$ only to objects that exist at $w$ ). This is important since the truth of the statements of necessity Kripke (correctly) embraces - Necessarily $\forall \boldsymbol{x}(\boldsymbol{A x} \supset \boldsymbol{B} \boldsymbol{x})$ and Necessarily $\forall \boldsymbol{x}(\boldsymbol{A} \boldsymbol{x} \leftrightarrow \boldsymbol{B} \boldsymbol{x})$ - cannot be derived from the truth of the unmodalized identification statements $-\forall \boldsymbol{x}(\boldsymbol{A} \boldsymbol{x} \supset \boldsymbol{B} \boldsymbol{x})$ and $\forall \boldsymbol{x}(\boldsymbol{A x} \leftrightarrow \boldsymbol{B} \boldsymbol{x})$ - plus characterizations of $A$ and $B$ as persistently essential. What follows from these premises is only the truth of weaker modal claims. To demonstrate the truth of the desired, stronger modal statements, one needs the full story given in Beyond Rigidity, which appeals to aspects of Kripke's analysis that go beyond both rigidity and essentiality. 
Let us focus on the question of whether the predicates be $a$ man and be human can apply at a circumstance to individuals who do not exist at that circumstance. In addressing this question, the crucial contrast is between (5), on the one hand, and examples like those in (6), on the other.

5. Plato is dead.

6a. *Plato is alive/*is a philosopher $/ *$ is Greek/* is a man/ *is human.

b. Plato was alive/was a philosopher/was Greek/was a man / was human.

Although we do say Plato is dead, we don't say Plato is a philosopher (except when speaking in the "specious present"). Instead we say Plato was a philosopher, and similarly for the other examples in (6), including Plato (*is) was a man, and Plato (*is) was human. The implications of this are significant. The truth of (5) is accounted for by the facts (i) that the name Plato now refers to Plato, even though Plato does not now exist, and (ii) that the predicate be dead (which arises from combining the adjective dead with the copula) now applies to Plato, even though Plato does not now exist. The falsity of the sentences in (6a) results from (i) plus the fact (iii) that the predicates be alive, be a philosopher, be Greek, be a man, and be human do not now apply to Plato (because Plato does not exist now), even though they did apply to him at certain times in the past when he did exist (as shown by the truth of the sentences in (6b)). Note in particular that this is true of the natural kind predicates be a man and be human, which are naturally understood as applying to any individual that they ever apply to at all (and only) the times at which that individual exists.

The corresponding point for modality is that these, and other typical members of the class of essentialist natural kind predicates, are persistently, rather than obstinately, essential. This is illustrated by the following fanciful example. ${ }^{2}$ If, having isolated a particular human sperm and egg, I name the individual

${ }^{2}$ See Salmon 1987, for discussion of cases of this sort. 
who would result from their union - under certain specified but never actually fulfilled conditions - Philo, then (7b) will be true, even though the sentences in (7a) are false.

7a. *Philo (actually) exists /*is (actually) a human being.

b. Philo would have existed, and would have been a human being, if certain conditions had been fulfilled.

Supposing, as seems natural, that the name Philo refers to Philo even with respect to possible world-states (including the actual world-state) at which he doesn't exist, we may conclude that the predicate is a human being does not apply to Philo with respect to world-states, like the actual world-state, in which he doesn't exist, even though it does apply to him with respect to all world-states in which he does exist. In short, is a human being is persistently, but not obstinately, essential. The same point holds for the other natural kind predicates in which GómezTorrente is interested.

Why, then, does he think otherwise? I believe it is because he has drawn the wrong lessons from his examples - here numbered (8) and (9).

8. Some men (human beings) are dead.

9. Some men (human beings) were prevented from developing when sperm $S_{1}$ and egg $E_{1}$, and sperm $S_{2}$ and egg $E_{2}$, did not unite, and went on to die.

Speaking about these examples, he says:

The first would seem true now, but under standard assumptions it is true at a time $t$ only if some object that is a man at $\boldsymbol{t}$ does not exist at $t$. The second would seem true (given suitable stipulations about the names it contains), even in the actual world, but under standard assumptions it is true at a world $w$ only if some object that is a man at $\boldsymbol{w}$ does not exist at $w$. (My emphasis.) 
Let us reconstruct the relevant reasoning: (8) is taken to have roughly the logical form (8a), where the quantifier - [Some $x$ : Man $x]$ — ranges over previously existing, as well as presently existing, individuals.

8a. [Some $x$ : Man $x$ ] Dead $x$

(8a), and hence (8), is taken to be true at the present time $t$, only if Dead $x$ is true at $t$ relative to some assignments of past and present individuals to $x$ which also make Man $x$ true at $t$. Since an assignment of an individual to $x$ can make Dead $x$ true at $t$ only if that individual existed prior to $t$, but no longer exists at $t$, the truth of (8a), and hence (8), requires the predicate Man to apply at $t$ to individuals who once existed, but no longer do at $t$. (9) is similar. Its logical form is taken to be something roughly along the lines of $(9 a)$.

9a. [Some $x$ : Man $x$ ] (x was prevented from developing when such and such occurred)

(9a), and hence (9), is judged to be true at the actual worldstate@, only if x was prevented from developing when such and such occurred is true relative to some assignments of possible individuals to $x$ which also make Man $x$ true at @. Since an assignment of an individual to $x$ can make $x$ was prevented from developing when such and such occurred true at @ only if that individual exists with respect to certain nearby world-states $w$ (in which such and such didn't occur), but doesn't exist with respect to@, the truth of (9a), and hence (9), requires the predicate Man to apply at @ to individuals who could have existed but don't. Generalizing, Gómez-Torrente concludes that the essentialist predicate Man (in the sense of one who is a human being) applies at an arbitrary world-state $w$ to all and only those individuals who are men (human beings) at any world state. In short, he concludes that predicates is a man and is a human being are obstinately, rather than persistently, essential.

That, I take it, is the reasoning behind Gómez-Torrente's remarks. Although the reasoning is seductive, it can't, I think, 
be right. One clue that it can't is provided by examples like those in $(10-13)$.

10. Some of my Princeton colleagues are dead.

11. Some widely respected philosophers are dead.

12. Some geniuses were prevented from developing when certain sperms and eggs carrying DNA ideally suited for intellectual ability did not unite, and went on to die.

13. Some catastrophes were prevented from occurring when park rangers put out wildfires caused by lightning.

First consider (10) and (11). Just as I could use Gómez-Torrente's (8) to say something true, so I could use (10) to assert a truth - even though I now have no Princeton colleagues. Since I am no longer at Princeton, no one is my Princeton colleague. To take one example, Gil Harman was my Princeton colleague, but he is not my Princeton colleague now. And, of course, David Lewis was also my Princeton colleague, though, in his case, he is no longer anyone's colleague, since he is dead. Similar remarks apply to (11). What these examples show is that the truths asserted at $t$ by certain uses of sentences of the form

\section{Some so and so's are dead.}

require not that the predicate is so and so applies at $\boldsymbol{t}$ to some individual who does not exist at $t$, but that the predicate previously applied to such an individual. Since Gómez-Torrente's example (8) is itself of this form, his use of it to assert a truth does not provide evidence that the predicates be a man, and be human now apply to individuals who do not exist. Rather, it provides evidence that these predicates once applied to individuals who, though they existed then, no longer do. Hence, it does not provide evidence that these natural kind predicates are obstinately essential.

Examples (12) and (13) extend this point to modality. Being a genius is not an essential property of a person who has it, and 
being a catastrophe is not an essential property of every catastrophic event. For example, Saul Kripke could have existed, even if something about his very early development had been slightly different, with the result that he was only very smart, rather than being the genius that he is. Similarly, the same burning of houses in a forest may be catastrophic in one possible circumstance, and not catastrophic in another (if, for example, in the latter, the houses were slated for destruction anyway, and the residents had been provided with better housing elsewhere). Hence the predicates, be a genius and be a catastrophe are not essentialist, let alone obstinately essentialist. Nevertheless, the examples in which they occur are on a par with GómezTorrente's (9). What is required by the truth of the propositions (12) and (13) are used to assert is not that there be individuals who are actually geniuses, while not existing, or events that are actually catastrophes, even though they never occur. What is required is that there be individuals who would have been geniuses, if they had existed, and events that would have been catastrophes, if they had occurred. A corresponding point holds for the proposition asserted by Gómez-Torrente's use of (9). Its truth requires not that there be individuals who are actually men (human beings), even though they don't exist, but that there be individuals who would have been men (human beings), if they had existed. Hence, the ability to use (9) to assert a truth provides no evidence that the predicates be a man, and be a human being apply at world-states to individuals who do not exist at those states. Since these predicates do express essential properties of individuals who have them, they are essentialist predicates. However, they are persistently essential - as is shown by examples like (6) - rather than obstinately essential. This conclusion holds quite generally for typical members of the subclass of natural kind predicates that express essential properties of the things to which they apply.

I will not here offer any formal analysis of the syntax and semantics of the sentences of the form (14) and (15) used to make this point. 
15. Some so and so's were prevented from developing when such and such occurred.

However, it is not hard to see what is going on in the uses of these sentences we have been considering. Consider again example (10) — Some of my Princeton colleagues are dead. Although the quantifier clause, some of my Princeton colleagues, does not contain any overt bearer of tense, the uses of (10) we have been considering are ones in which a certain time reference is understood. What is said in these uses is roughly that which is expressed by (10a).

10a. Some of my previous Princeton colleagues are dead.

Similar remarks apply to (12) - Some geniuses were prevented from developing when certain sperms and eggs carrying DNA ideally suited for intellectual ability did not unite, and went on to die. Although the quantifier clause, some geniuses, does not contain any modal operator, what is said by the uses of (12) we have been considering is roughly that which is expressed by $(12 a)$.

12a. Some individuals who would have been geniuses were prevented from developing when certain sperms and eggs carrying DNA ideally suited for intellectual ability did not unite, and went on to die.

There are different ways of formally capturing the force of examples like these. However, since deciding among them would raise questions irrelevant to our main concerns, we need not do so here. The important point for us is that the truth of examples of the form (14) and (15) don't require the predicates is so and so to now, or actually, apply to individuals that do not now, or actually, exist.

This, I believe, sinks the strategy of vindicating Kripke's doctrine that typical a posteriori statements of theoretical identification -including universally quantified conditionals and biconditionals involving natural kind predicates - are necessary, if true, by defining rigidity for predicates as essentiality, and 
appealing to the rigidity of the relevant predicates. Although a number of the natural kind predicates in question are, indeed, rigid in this special sense, they are not obstinately rigid (i.e. essential). As a result, one cannot derive the truth of the necessitations of the original identification statements. Since these strong statements of necessity — rather than weaker statements of necessity corresponding to weaker notions of essentialityare the ones that Kripke, rightly, wishes to explain the truth of, appealing to rigidity as essentiality won't do the job. As indicated earlier, this does not mean that Kripke's doctrine can't be vindicated (in many cases). It can. However, in order to do so, one must invoke aspects of his model that go beyond rigidity and essentiality.

This brings me to my final point of disagreement with Gómez-Torrente. Unlike him, I do not believe that the notion of rigid designation for general terms, or predicates containing them, should be defined in terms of essentiality. There are two cases to consider. The first involves predicates - like is red and is human. It is, I think, central to the idea of rigid designation that it involve sameness of extension across different circumstances of evaluation. Hence, if the notion of rigidity is to be extended to predicates, such predicates should have the same extensions across different circumstances in which they have extensions at all. Although obstinately essential predicates satisfy this condition, nonessential predicates, and predicates that are more weakly essential, do not. Since so few natural kind predicates are obstinately essential, extending the notion of rigid designation in this way is of little theoretical interest.

The next case to consider is that of general terms. When writing Beyond Rigidity, I did not, when discussing rigidity, clearly distinguish general terms, like cow and tiger, from predicates, like is a cow and is a tiger, that contain them - a practice which, as we have seen, Naming and Necessity is not entirely free of.

According to the view I advocate, then, terms for natural kinds are much closer to proper names than is ordinarily supposed. The old term 'common name' is thus quite appropriate for predicates 
marking out species or natural kinds, such as 'cow' or 'tiger' [...]. (p. 127; my boldface emphasis.)

Prodded by Bernard Linsky (forthcoming) and Nathan Salmon (2003), I have come to see this as an error. I now think that there is a natural way of extending Kripke's distinction between rigid and nonrigid designators from singular to general terms - even though it does not extend to predicates constructed from those terms, and so does not provide an explanation of the necessity of true statements of theoretical identification involving natural kind predicates. ${ }^{3}$

In the case of both singular and general terms, for an expression to rigidly designate its extension is for it to designate the same extension with respect to every possible world-state (in which it has an extension at all). On this account, simple natural kind terms like water, gold, electricity, red, and tiger - as well as ordinary general terms like bachelor, philosopher, automobile and triangle - designate the same extension with respect to each world-state. As I see it, however, the two differ importantly in the metaphysics of their designata. Whereas a term like bachelor designates a property that may be distinguished from other properties that are necessarily coextensive with it, natural kinds differ from one another only if there are possible worldstates in which some of their instances are different. Intuitively this seems plausible; it is hard to imagine two distinct species of animal, two distinct substances, or two distinct colors which have precisely the same instances in every possible world-state. This is important for my linguistic model. Consider, for example, the color red. Color science tells us that the object-color red is determined by a certain type of surface spectral reflectance property - one which specifies proportions of light reflected at different wavelengths. Let $Q$ be a complex general term of English explicitly mentioning proportions of light reflected at different wavelengths that designates this property. The term red is clearly not synonymous with $Q$. The same can be said

${ }^{3}$ See my "Reply to Critics", in the forthcoming symposium on Beyond Rigidity, in Philosophical Studies. 
for other descriptive general terms. Suppose there is a further complex term $Q^{\prime}$ that applies to surfaces on the basis of a specification of their minute physical structure, which turns out to be necessarily equivalent to $Q$. Then, although red is necessarily equivalent to both $Q$ and $Q^{\prime}$, it is synonymous with neither. The different complex properties designated by these general terms have equal claim to determining the color red, but neither is identical with the color itself. What is said here about red, and the color it designates, applies to many linguistically simple general terms for natural kinds, and their designata.

Although most of this picture can be found in Beyond Rigidity, one important distinction was left out - the distinction between a general term, like red, and the predicate is (or be) red formed from it by attaching the copula. Whereas red directly and rigidly designates the color red — which is both its extension and semantic content - the predicate is red designates the set of individuals to which it applies, which is its extension. Since the set of red things varies from world-state to world-state, there is no natural extension of Kripke's notion of rigid designation to predicates that classifies is red as rigid, even though the general term red is rigid in roughly Kripke's original sense. Similar remarks apply to other general terms for natural kinds and the predicates that contain them - as well as to ordinary general terms like bachelor, and the predicates that result from combining them with the copula. The semantic content of such a predicate is a complex, consisting of the semantic content of its general term plus the content of the copula (being an instance of). For each world-state $w$, the latter assigns to the argument provided by the semantic content of the general term at $w$ the class of instances of that argument. Since red is directly referential, its semantic content is the color itself, which is the argument it provides to the content of the copula at every world-state. Thus, for all intents and purposes, the semantic content of the predicate is red may be taken to be the property of being (an instance of the color) red.

Next consider the predicate is the color of stop signs. Following the lead of Linsky and Salmon, we may treat the de- 
scription it contains as a general term, the F: F(stop signs) \& Color $(\boldsymbol{F})$, in which $F$ is a second-order predicate-variable ranging over kinds of individuals (where stop signs are taken to be individuals), and Color is a higher-order predicate of kinds. Unlike the simple general term red, the description (which is a complex general term) is nonrigid, denoting the color red at the actual world-state, while denoting other colors at different possible world-states. When combined with the copula, the description forms a predicate the semantic content of which is the complex $<$ being an instance of, $D>$, where $D$ is the semantic content of the description. Since $D$ determines the color red at the actual world-state, the actual extension of the predicate is the set of red things, whereas its extensions at other world-states may be sets of things of different colors. The end result is a distinction between rigid and nonrigid general terms that is a helpful addition to, and correction of, Beyond Rigidity. However, it does not significantly affect the account given there of the necessity of true, a posteriori statements of theoretical identification involving natural kind predicates.

\section{Reply to Ezcurdia}

Whereas Gómez-Torrente focuses on my account of natural kind terms, and predicates containing them, Ezcurdia focuses on my account of names and indexicals, with special emphasis on their behavior in the content clauses of propositional attitude ascriptions. Although she is sympathetic to my claims BR1-BR3, and may even be prepared to accept them, she emphatically rejects BR4. ${ }^{4}$

${ }^{4}$ After arguing vigorously against BR4, she writes, "nothing of what has been argued here challenges the general Millian-Russellian approach that Soames advocates nor in particular the arguments that he gives in favour of the Millian character of names. Names may still only contribute their referents to the propositions expressed by (utterances of) sentences containing them, and propositions are still made up of references structured in a certain way. Secondly, nothing of what has been argued here challenges the account given by Soames of the epistemological difference between (3) [Hesperus is Phosphorus] and (4) [Hesperus is Hesperus] nor his general approach to distinguishing amongst the propositions that are merely asserted and those that 
BR1. Names, indexicals, and variables are directly referential -i.e. the semantic content of such a term (its contribution to propositions semantically expressed by sentences containing it), relative to a context $C$ and assignment $A$, is simply its referent relative to $C$ and $A$.

BR2. Simple sentences — such as Hesperus is Hesperus and Hesperus is Phosphorus - which differ only in the substitution of coreferential names or indexicals (relative to appropriate contexts) semantically express the same (Russellian) propositions.

BR3. Pairs of sentences of the sort mentioned in BR2 may differ in "cognitive value," even though their semantic contents (i.e. the propositions they semantically express) are the same. This difference is explained by pragmatic differences in the propositions they are used to entertain, assert and convey.

BR4. Attitude ascriptions - such as Jones asserted/believed that Hesperus is Hesperus and Jones asserted/ believed that Hesperus is Phosphorus - which differ only in the substitution of coreferential names, semantically express propositions which (a) report beliefs in the same (singular, Russellian) proposition (semantically expressed by their complement clauses), and (b) cannot differ in truth value.

The basis for Ezcurdia's rejection of BR4 lies in a cluster of views she holds about (i) the explanatory uses to which belief, and certain other attitude, ascriptions are put, (ii) the centrality of these uses in our understanding of what belief and certain other attitudes are, and (iii) the relationship between failures of substitutivity in belief, and certain other attitude, ascriptions and the ability of those ascriptions to play the explanatory roles that (in her view) are constitutive of their meanings.

are also semantically expressed. I think Soames's account of the difference that may emerge between (3) and (4) is correct." 
In a moment, I will turn to these views. Before I do, however, I will highlight a tension in the overall view to which Ezcurdia seems to incline. There is an inherent difficulty in combining an acceptance of BRl-BR3, to which she inclines, with a rejection of $\mathrm{BR} 4$, on which she insists. As the following argument indicates, $\mathrm{Pl}$, which is a corollary of BRl-BR3, leads very naturally to $\mathrm{C} 4$, which may naturally be taken to conflict with the rejection of BR4.

P1. Hesperus is Hesperus and Hesperus is Phosphorus semantically express the same proposition.

P2. The proposition semantically expressed by Hesperus is Hesperus is the proposition that Hesperus is Hesperus. The proposition semantically expressed by Hesperus is Phosphorus is the proposition that Hesperus is Phosphorus.

P3. For any propositions $p$ and $q$, and agent $x$, if $p=q$, then $x$ asserts / believes $p$ iff $x$ asserts / believes $q$.

C1. Hence (from P2 and P3), for any agent $x, x$ asserts / believes the proposition semantically expressed by Hesperus is Hesperus iff $x$ asserts / believes the proposition that Hesperus is Hesperus. (Ditto for Hesperus is Phosphorus.)

C2. Similarly (from P1 and P3), for any agent $x, x$ asserts / believes the proposition semantically expressed by Hesperus is Hesperus iff $x$ asserts / believes the proposition semantically expressed by Hesperus is Phosphorus.

C3. So (from C1 and C2), for any agent $x, x$ asserts / believes the proposition that Hesperus is Hesperus iff $x$ asserts / believes the proposition that Hesperus is Phosphorus.

P4. But surely, for any agent $x, x$ asserts / believes the proposition that Hesperus is Hesperus (or the proposition that Hesperus is Phosphorus) iff $x$ asserts / believes that Hesperus is Hesperus (or that Hesperus is Phosphorus). 
C4. Therefore, for any agent $x, x$ asserts / believes that Hesperus is Hesperus iff $x$ asserts / believes that Hesperus is Phosphorus.

Although Ezcurdia expresses considerable sympathy for P1, she firmly rejects C4. However, since the argument is valid, and $\mathrm{P} 2$, P3, and $\mathrm{P} 4$ are all but undeniable, it is not clear that one can reasonably accept $\mathrm{Pl}$ while unequivocally rejecting $\mathrm{C} 4$. Although Ezcurdia might see this as reason for tempering her enthusiasm for P1, I would urge her to look at things the other way around. Given the strong case that has been made for P1, one should think again about C4.

If the view presented in chapters 3-8 of Beyond Rigidity is correct, then there is a way of accepting the argument, and with it C4, while respecting the intuition that attitude ascriptions which differ solely in the substitution of coreferential names (or indexicals) are not cognitively or conversationally equivalent. On the view defended there, the propositions semantically expressed by such ascriptions cannot differ in truth value. Nevertheless, assertive utterances of the ascriptions may express (and be used to communicate) different beliefs of the speaker, and result in assertions with different truth values that share the contents of those beliefs. For example, an assertive utterance of

16. Jones asserted / believed that Hesperus was Phosphorus in a conversational situation in which different descriptive information is (pragmatically) associated with the two names by speakers and hearers may, in certain cases, express a false belief about Jones, and result in a false assertion that shares the content of that belief, even if an utterance of the semantically equivalent ascription

\section{Jones asserted / believed that Hesperus was Hesperus}

in the same situation would express only a true belief about Jones, and result in no false assertion whatsoever.

Let us suppose that CS is such a conversational situation. In CS, I would not be willing to assertively utter (16), even though 
I would be willing to assertively utter both (17) and the negation of (16). How, then, one might ask, can I accept the argument for C4? I accept it in the sense that I recognize (i) that the propositions semantically expressed by the premises $\mathrm{P} 1-\mathrm{P} 4$ are true, (ii) that C4 is a logical consequence of those premises, and (iii) that the proposition semantically expressed by $\mathrm{C} 4$ is both a necessary and an a priori consequence of the propositions the premises semantically express. In possible conversational situations in which I use the sentences in the argument to assert simply the propositions they semantically express, I am correct when I assertively utter both premises and conclusions.

However, these are not the only possible, or even the most natural, conversational situations. If CS* is a situation in which I use the clauses that Hesperus is Phosphorus and that Hesperus is Hesperus in attitude ascriptions to make assertions about the propositional attitudes that agents bear to different descriptive enrichments of the propositions the clauses semantically denote, then I will reject C4 in CS*. If, in addition, my use of that-clauses in $\mathrm{CS}^{*}$ to pick out descriptive enrichments of the propositions they semantically denote is consistent throughout the premises and conclusions of the argument, I will, of course, also reject P2 - since on any such consistent use, utterances of the sentences in P2 will result in false assertions.

Finally, there are possible conversational situations in which I use the that-clauses to pick out changes as the conversation progresses (just as there are conversations in which I use he to denote changes from one moment to the next). If I use the that-clauses in $\mathrm{P} 2$ to pick out the propositions they semantically denote, and nothing else, while using them in C3 and C4. to pick out descriptive enrichments of those propositions, then I may affirm the premises of the argument while denying its ultimate conclusions, without committing any logical error. Written down, and taken out of context, the words I use in doing this may appear illogical, but that is only because crucial contextual information has been lost. The original conversational situation was coherent (even if potentially misleading). 
The virtue of this account is that it allows us to explain and simultaneously embrace (i) the persuasive case that can be made for the truth of $\mathrm{Pl}$, (ii) the powerful intuition that there is a natural and straightforward sense in which the argument from $\mathrm{P} 1-\mathrm{P} 4$ to $\mathrm{C} 4$ is sound, if $\mathrm{Pl}$ is true, and (iii) the equally powerful intuition that there is a natural and straightforward sense in which C4 can, and should, be rejected. The fact that each of (i)-(iii) is highly plausible makes the account attractive. The fact that it is not easy to combine them into a coherent and consistent whole makes the virtue of the account difficult to duplicate. Since Ezcurdia rejects crucial aspects of the Beyond Rigidity treatment of the semantics and pragmatics of attitude ascriptions, she cannot endorse the account of (i)-(iii) given here. Nor, as far as I can tell, does she have another way of doing justice to all three. In my opinion, this provides one reason for doubting the correctness of her critique.

The heart of that critique is an alleged connection between what is required to competently use and understand belief (and certain other propositional attitude) predicates, on the one hand, and the possibility of changes in truth value arising from substitution of coreferential names or indexicals in the content clauses of attitude ascriptions containing such predicates, on the other. According to Ezcurdia, the connection between the two is provided by the role of belief (and some other) attitude ascriptions in explaining actions and mental states. Her account of competence with these ascriptions begins with the observation that such competence brings with it a recognition that they report relations - believing, knowing, realizing, etc.- that agents bear to the referents of that-clauses. In addition, she suggests, in order to know what these relations are -in the minimal sense required to understand attitude ascriptions involving themone must know that such ascriptions are often used to explain the actions and mental states of those agents. As examples, she gives the explanations provided by (18)-(21).

18. I stopped looking for the shopper who was spilling the sugar throughout the supermarket because I realized that I was the one spilling the sugar throughout the supermarket. 
19. Lois Lane did not go with Clark Kent because she believed that Clark Kent was not Superman.

20. Although Peter wanted to see Hesperus, he did not wake up early in the morning to see it, because he believed that Hesperus is not visible in the morning.

21. Paul came to believe that Carl Hempel wrote Aspects of Scientific Explanation because his supervisor told him that he did.

Let us suppose, for the sake of argument, that Ezcurdia is right about this: competence with certain attitude predicates involves recognition of the possibility of using them to give explanations like these. What does this have to do with whether substitution of coreferential names and indexicals ever changes truth values of propositions semantically expressed by attitude ascriptions containing such predicates? Ezcurdia never really tells us. Instead, she simply notes that in certain cases in which propositional attitude ascriptions are used in explanations, substitution would result in a loss of explanatory power.

For a competent speaker of the language to use propositional attitude sentences in explanations of actions or mental states, she must be sensitive to the changes that can be brought about by allowing for substitutions of coreferential terms such as 'Clark Kent' for 'Superman' in the embedded clause in [19]. The speaker competent in attitude reports must know that substituting one name for the other just loses the explanatory power of the belief report in [19]. Similarly, substituting 'Maite' in the 'that'-clause in [18] when uttered by me [Maite Ezcurdia] for ' $\mathrm{I}$ ' as in [18'] would make the attitude report lose its explanatory power.

$18^{\prime}$. I stopped looking for the shopper who was spilling the sugar throughout the supermarket because I realized that Maite was the one spilling the sugar throughout the supermarket.

But more than this. Assuming that I am amnesiac and I do not know that my name is 'Maite', [18'] would be false whereas [18] would be true. (Ezcurdia 2004, pp. 71-72 in this issue.) 
Although what is said here is mostly unobjectionable, an essential distinction has been left out. The distinction is between the effects of substitution on the explanations provided by the propositions asserted by relevant utterances of (18)-(21) and the effects of substitution on the explanations provided by the propositions these sentences semantically express. Since this distinction is crucial to the pragmatic and semantic framework for treating attitude ascriptions in Beyond Rigidity, Ezcurdia's remarks do not succeed in discrediting it. In particular, they do not succeed in showing that the conditions required for competence with attitude ascriptions are inconsistent with a guarantee that substitution of coreferential names or indexicals in the content clauses of such reports preserves the truth values of the propositions semantically expressed - even if such competence does require speakers to recognize that attitude ascriptions are often used in giving explanations of actions and mental states.

In what follows, I will try to explain in more detail why this is so. If I am right, then the basic framework of Beyond Rigidity can accommodate the legitimate insights behind Ezcurdia's critique. As we will see, however, the most effective way of doing this requires taking advantage of an independently motivated revision of one important part of that framework. It is in connection with this revision that Ezcurdia's observations about explanation can be used to make a solid critical point.

The centerpiece of the first eight chapters of Beyond Rigidity is the framework it provides for understanding how the proposition semantically expressed by a sentence $S$ (a heavily theoretical notion) is related to the propositions $S$ is used to assert and convey in different normal contexts of utterance (a pragmatic notion which is much more directly accessible to the intuitions of competent language users). Within this framework - which Ezcurdia, for the most part, accepts - showing that an ascription A believes that ...n... is subject to the sort of substitution failure sufficient to make her point requires showing that it is not the case that the proposition semantically expressed by the ascription is the proposition $p$ which consists simply of the attribution of the belief relation to the pair con- 
sisting of the referent of $A$ and the bare, Russellian proposition semantically expressed by ...x...., relative to an assignment of the referent of $n$ to ' $x$ '. In order to do this, she needs to demonstrate either (i) or (ii).

(i) It is not the case that $p$ is asserted by assertive utterances of the ascription by competent speakers in all relevant "normal" contexts.

(ii) There is some other proposition $q$ which satisfies the negation of (i) that is a better candidate for being the semantic content of the attitude ascription than $p$ is.

Of course, not all utterances of A believes that ...n... will occur as parts of attempted explanations of actions and mental states. Hence, if there is reason to believe that all these (nonexplanatory) utterances result in assertions of $p$ (along, perhaps, with assertions of various other, descriptively enriched, propositions in different contexts), then those reasons will remain in force - as will the reasons for thinking that no other, descriptively enriched, propositions are asserted in all these contexts-, in which case none of those propositions will remain in contention for qualifying as the semantic content of the ascription.

What about utterances of belief ascriptions that do occur as parts of explanations of actions and mental states? Take, for example, (21). Suppose that Mary assertively utters it in order to explain why Paul believes what he does. According to Beyond Rigidity, there are two cases to consider - (i) those in which the proposition asserted is simply the bare Russellian proposition $p$ expressed by (2la), relative to an assignment of Mr. Hempel to ' $x$ ', and (ii) those in which Mary asserts some descriptive enrichment $p^{+}$of $p$, indicated by $(21 \mathrm{~b})$, relative to a similar assignment. ${ }^{5}$

21a. Paul came to believe that $x$ wrote Aspects of Scientific Explanation because his supervisor told him that $x$ did.

${ }^{5} \mathrm{I}$ put aside issues raised by the anaphora in the subordinate clause. 
21b. Paul came to believe that [the $y: D y \& y=x$ ] ( $y$ wrote Aspects of Scientific Explanation) because his supervisor told him that [the $y: D y \& y=x$ ] did.

Case (i) poses no problem, since the proposition asserted is simply the proposition semantically expressed. In this case, a certain de re belief of Paul is explained by citing how he acquired it from his supervisor. Surely, the competence conditions for belief ascriptions allow their use in such explanations.

Case (ii) is trickier. Here, the bare, Russellian proposition $p$, expressed by (2la) relative to an assignment of Mr. Hempel to ' $x$ ' - and identified in Beyond Rigidity as the proposition semantically expressed by (2l) - is not a necessary and a priori consequence of the descriptively enriched proposition $p+$ that Mary asserts. (Proposition $p+$ can be true and $p$ false in a situation in which Paul knows, of Mr. Hempel, prior to speaking to his supervisor, both that Mr. Hempel wrote Aspects of Scientific Explanation and that Mr. Hempel "was $D$ " - without knowing, or being in a position to draw the conclusion, that the author of the book "was $D$ ".) Thus, the assertion of $p+$ does not, in itself, guarantee the simultaneous assertion of $p$. Although I recognized this in Beyond Rigidity, I thought I had an independent argument that $p$ must also be asserted. ${ }^{6}$ As I explain in "Naming and Asserting", I have now come to think otherwise. ${ }^{7}$ Proposition $p$ need not be asserted by Mary in the situation imagined - in which case $p$ does not satisfy the condition in Beyond Rigidity for being the proposition semantically expressed by (21). Since no other proposition does either, that condition must be revised.

That was the burden of "Naming and Asserting". According to the new, revised view, what is necessary in order for a proposition $q$ to be semantically expressed by a sentence $S$ is that assertive utterances of $S$ by competent speakers in "normal" contexts always result in the assertion either of $q$, or of some descriptive enrichment of $q$ (or both). On this view, the proposition semantically expressed by our example (21) remains the

${ }^{6}$ See the discussion of example (38) on pages $230-235$.

${ }^{7}$ See Soames 2005. 
bare Russellian proposition $p$ - just as before. However, now the fact that Mary asserts only $p^{+}$, and not $p$, is consistent with the fact that $p$ is semantically expressed by the sentence she utters.

This is the key to understanding failures of substitutivity. When a speaker assertively utters (21) in a situation in which substitution for Carl Hempel would result in a change in truth value, that change is in the proposition asserted, not the proposition semantically expressed. In such a situation, the speaker asserts a descriptive enrichment $p+$ of the proposition $p$ that is semantically expressed by (21). Had the speaker assertively uttered a sentence differing from (21) only in the substitution of a coreferential name or indexical for Carl Hempel, then the same proposition $p$ would have been semantically expressed, but a different proposition - either $p$ itself or a different descriptive enrichment $p++$ of $p$ - would have been asserted (if the descriptive information associated with Carl Hempel in the context differs from the information, if any, associated with the name or indexical substituted for it). If $p+$ is true and $p$ and $p++$ are false, then we have the sort of failure of substitution to preserve truth value that, I believe, Ezcurdia had in mind. Although the original system of Beyond Rigidity allows for this sort of case, it (wrongly) maintains that the true assertion of $p^{+}$is invariably accompanied by the false assertion of $p$ - and hence that the speaker who assertively utters (21) both says something true and says something false. This, I suspect, is something that Ezcurdia would (rightly) reject. If so, then the critical point she raises does tell against the specific formulations in Beyond Rigidity, though not against the general framework presented there, or the version of that framework advanced in "Naming and Asserting".

Similar points hold for her other examples. Save for one peculiarity, examples (19) and (20) are like (21) in all relevant respects. The peculiarity involves the name Superman in (19). As indicated in Beyond Rigidity, Superman (as used in the fiction) is a rare example of a syntactically simple proper name that is so widely associated with the same simple description 
-roughly, man with superhuman powers - that it is reasonable to suppose that it is what I call a partially descriptive name. ${ }^{8}$ If it is, then its semantic content is that of the description the $\mathrm{x}$ : $\mathrm{x}$ is a man and $\mathrm{x}$ has superhuman powers and $\mathrm{x}=\mathrm{y}$, relative to an assignment of the superhero himself to ' $y$ '. Because of this, substitution of another coreferential name for it in attitude ascriptions will sometimes change not just the proposition asserted by an utterance of the ascription, but also the proposition semantically expressed. All of this is fully compatible with both the original formulation of my framework in Beyond Rigidity and the revised formulation in "Naming and Asserting". Because of this, examples containing the name Superman are not suitable for making Ezcurdia's critical point.

Example (18) is special in a different way. ${ }^{9}$ As with (20) and (21), it can be used to assert simply the proposition it semantically expresses, in which case the content of the mental state figuring in the explanation is the bare, Russellian proposition semantically expressed by the content clause of the attitude ascription. In a case like this, what Maite asserts by uttering (18) is that she stopped looking for the messy shopper because she realized, of herself, that she was that shopper -i.e. because she came to bear the relation of realizing-true to the singular proposition that attributes to her the property of being the one spilling the sugar. In what sense does this provide an explanation for her action? Let us keep things simple by supposing that it purports to explain the action by citing a sufficient cause of it (while leaving aside whatever else may be required to provide a justifying reason for the action). Adopting this simplifying assumption, and putting unnecessary complications aside, we may identify the truth conditions of the explanation as consisting, roughly, of (i) and (ii): (i) that Maite did stop her search after coming to believe, at certain time $t$, of herself, that she was the one spilling the sugar, and (ii) that had she not come to believe this at $t$, she would not have stopped then (unless the example was one of either causal preemption or overdetermi-

\footnotetext{
${ }^{8}$ Beyond Rigidity, pp. 121-122.

${ }^{9}$ The example comes from Perry 1979.
} 
nation). ${ }^{10}$ Clearly, an explanation of this sort - given by an assertive utterance of (18) - might turn out to be true; and if it did, this truth would be preserved by a substitution of 'Maite' for ' $\mathrm{I}$ ' in the complement of realize - whether or not Maite knew that her name was 'Maite'. ${ }^{11}$ Thus, the use of the attitude ascription in (18) to give explanations of actions is compatible with the semantic and pragmatic analysis of such ascriptions given in Beyond Rigidity.

But what about the case in which (i) Maite is pushing her cart in the supermarket following a trail of sugar that she wrongly thinks is coming from someone else's cart, (ii) she hears an announcement over the loudspeaker, "Maite Ezcurdia, please stop where you are. You are spilling sugar off your cart", but (iii) she does not stop because she has amnesia and has forgotten that her name is 'Maite Ezcurdia'? In such a situation, she may continue her search, even after she comes to believe - on the basis of the announcement - the singular, Russellian proposition attributing to her the property of being the one spilling the sugar. She may keep on searching until she says to herself "I am the one spilling the sugar". Finally, in explaining her actions later, she might assertively utter (18), just as she did in the original case. However, in this case, what caused her to stop searching was not her coming to believe the singular proposition that attributes to her the property of being the one spilling the sugar. Thus, the proposition which, on my analy-

${ }^{10}$ Roughly speaking, we may take the content of (ii) as holding iff of all the possible world-states in which it is not the case that Maite comes to believe the relevant singular proposition (and to realize that it is true) at $t$, those that are most similar to the actual world-state are those in which she continues searching at $t$.

${ }^{11}$ That is, the proposition semantically expressed by both (18) and (18') would be true, and would provide an explanation of Maite's action. In cases in which assertive utterances of these sentences resulted in the assertion of simply this proposition, the explanatory force of the asserted explanations would, of course, not be affected by the substitution. However, when one of the assertive utterances results in the assertion of a descriptive enrichment of the proposition semantically expressed that is not asserted by an utterance of the other sentence, substitution may affect the truth values of the asserted explanations, as in our discussion of (21). 
sis, is semantically expressed by both (18) and (18') (in which 'Maite' is substituted for 'I' in the complement of realize) is false - when taken as a description of this scenario.

What shall we say about this version of the case? One temptation to be resisted is to suppose that it invalidates our verdict about the previous case (in which Maite doesn't have amnesia, and she hears nothing over the loudspeaker). This temptation arises from the thought that what caused her to stop searching is the same in both cases - namely her coming to sincerely endorse the sentence (22), or, as we might put it, to accept its Kaplanian character.

\section{I am the one spilling the sugar.}

Since in the second (amnesia) case this did not involve coming (only then) to believe, or realize the truth of, the Russellian proposition which it semantically expresses, it might seem that coming to have this belief or realization could not have caused her action in the first case either. But then, the proposition semantically expressed by (18) cannot have provided the correct explanation of Maite's action, even in the first case.

To reason in this way is to make a mistake. To be sure, Maite's coming to accept the character of (22) caused her to stop searching for the messy shopper in both cases. In the first case, her coming to accept this character was her coming to believe (for the first time) the Russellian proposition it semantically expresses. Because of this, her coming to have that belief (bear that realization relation) was a correct explanation of her action in the first scenario, and the proposition semantically expressed by (18) was true. The reason it is not the correct explanation for her action in the second (amnesia) scenario is that in the new case she already has the relevant belief / realization prior to her coming to accept the character of (22). Hence, her coming to believe the proposition it semantically expresses — which is the proposition semantically expressed by the complement of realize in (18) - does not explain her action in that case.

Is there some other proposition which (i) she comes to believe, or realize to be true, as a result of her coming to accept 
the character of (22), and (ii) is such that her coming to believe that proposition, or realize it to be true, does explain her action in the amnesia case? In principle, there could be. Perhaps in saying to herself I am the one spilling the sugar she was thinking of herself as the one pushing this cart (accompanied by an appropriate demonstration) - in which case, she may have been using (22) to entertain not just the bare Russellian proposition it semantically expresses, but also the descriptive enrichment of that proposition expressed by $(22+)$.

$22+$. I, the one pushing this cart, am the one spilling the sugar (accompanied by an appropriate demonstration).

If so, then Maite's acceptance of the character of (22) in this situation may indicate belief in both the bare Russellian proposition it semantically expresses and the descriptive enrichment of that proposition expressed by $(22+)$. In the situation as we are now imagining it, her coming to believe the latter proposition, and realize it to be true, does cause her action. Finally, let us suppose that she continues to think of herself in the same way afterwards, and that when she assertively utters (18), she asserts and conveys the descriptive enrichment - expressed by (18+) of the proposition semantically expressed by the sentence she uttered.

18+. I stopped looking for the shopper who was spilling the sugar throughout the supermarket because I realized that I, the one pushing this cart, was the one spilling the sugar throughout the supermarket.

Given all this, we may conclude that the proposition asserted and conveyed by her utterance of (18) is true, and explains her action, even though (i) the proposition semantically expressed by (18) is false, and (ii) the proposition that would be asserted by Maite if she were to utter $\left(18^{\prime}\right)$ - in which 'Maite' is substituted for 'I' in the scope of 'realized' - would be false. All of this is fully compatible with the semantic and pragmatic account of attitude ascriptions developed in Beyond Rigidity and "Naming and Asserting". 
Of course, I haven't considered every case, and nothing guarantees that every version of the amnesia scenario can be handled in the manner just illustrated. For example, consider an expanded version of the case in which Maite not only hears the announcement on the loudspeaker, but also sees a reflection of herself pushing her cart in one of those distorting mirrors that are sometimes placed at the end of aisles to deter theft. Looking at the reflection, she says to herself That person, pushing that cart, is the one spilling the sugar, without recognizing herself or her cart. As a result, she comes to have the belief appealed to in the previous version of the case without, in the new version, coming to accept the character of (22), and without stopping her search for the messy shopper. Although this doesn't undermine the explanation given of the previous version of the case, it does prevent an identical explanation from being given for the new, expanded version. Perhaps we can find still another proposition which (i) Maite comes to believe when she accepts the character of (22), and (ii) is such that her realization that it is true does explain her action. But even if we can, it seems likely that we could then construct an even more elaborate version of the amnesia case in which appeal to belief in the new proposition wouldn't play the desired explanatory role.

In light of this, it is not implausible to suppose that there are some scenarios in which Maite's action can't be explained by her coming to realize the truth of any proposition the truth of which she had not already been aware of. In such a scenario, as in the earlier cases, her action can be explained by her coming to be in a state of mind that would lead her to endorse (22), and accept its character. However, in extreme scenarios of the sort now imagined, her coming to be in this state of mind does not coincide with her acquiring any new belief. If, in such a scenario, she assertively utters (18) in an attempt to explain her action, neither the proposition $p$ it semantically expresses (on my analysis), nor any descriptive enrichment of $p$ that may be asserted by the utterance will be true. Still, there may seem to be something right about the utterance. Somehow, it conveys the information that the speaker's action was caused 
by her being in a state of mind that would prompt acceptance of the character of the complement of realize, rather than by her believing the proposition expressed by that clause.

How this comes about is an open and controversial question. Some may hold that attitude ascriptions of the form (23) have special de se semantic readings, not shared by attitude ascriptions generally, in which the attitude verb reports a relation to the character of its complement, rather than to the content of that clause (as it normally does). ${ }^{12}$

23. I believe / know / realize, etc. that ... I. . .

Others may hold that there are no such special semantically de se readings, but that the relevant de se suggestions arise pragmatically. ${ }^{13}$ I incline to the latter view -in part because the former seems to lead to unacceptable differences in the semantically determined truth values of (23) and (24), in some cases in which $A$ and ' $\mathrm{I}$ ' are coreferential.

24. $A$ believes / knows / realizes that ... I. . .

However, no matter who is right about this, the de se problem is, I believe, a special, circumscribed one - over and above the problem of giving correct semantic and pragmatic treatments of the great mass of ordinary attitude ascriptions. Because of this, it does not provide a substantial basis for evaluating the framework for analyzing such ascriptions developed in Beyond Rigidity and "Naming and Asserting". Certainly, more needs to be said in order to deal with de se cases completely and correctly. As far as I can tell, however, whatever needs to be said can be incorporated into the framework given there.

\section{REFERENCES}

Boer, Steven and William Lycan, 1980, "Who Me?", The Philosophical Review, vol. 89.

${ }^{12}$ For an early, path-breaking discussion of de se attitudes, plus his own unique semantic analysis, see Lewis 1979. See also Chisholm 1981. For earlier discussions, see Castañeda 1966, 1967, 1968a and 1968b.

${ }^{13}$ See, for example, Boer and Lycan 1980. 
Castañeda, Hector, 1968a, "On the Phenomeno-Logic of the I", Proceedings of the XIVth International Congress of Philosophy. 1968b, "On the Logic of Attributions of Self-Knowledge to Others", Journal of Philosophy, vol. 65.

_, 1967, "Indicators and Quasi-Indicators", American Philosophical Quarterly, vol. 4.

- 1966, "He': A Study in the Logic of Self-Consciousness", Ratio, vol. 8.

Chisholm, Roderick, 1981, The First Person, University of Minnesota Press, Minneapolis.

Kripke, Saul, 1980, Naming and Necessity, Harvard University Press, Cambridge, Mass. (Originally published in 1972.)

Lewis, David, 1979, "Attitudes De Dicto and De Se", Philosophical Review, vol. 88, 1979. (Reprinted in his Philosophical Papers: Volume 1, Oxford University Press, Oxford/New York.)

Linsky, Bernard (forthcoming), "General Terms as Rigid Designators", forthcoming in a symposium on Beyond Rigidity in Philosophical Studies.

Perry, John, 1979, "The Problem of the Essential Indexical", Noûs, vol. 13.

Salmon, Nathan, 2003, "Naming, Necessity, and Beyond", Mind, vol. 112.

—_, 1987, "Existence", in J. Tomberlin (ed.), Philosophical Perspectives, Volume 1: Metaphysics, Ridgeview Press, Atascadero.

Soames, Scott, 2005, "Naming and Asserting", in Zoltan Szabo (ed.), Semantics vs. Pragmatics, Oxford University Press, Oxford/New York.

_ 2002, Beyond Rigidity, Oxford University Press, Oxford/New York.

- (forthcoming), "Reply to Critics", forthcoming in a symposium on Beyond Rigidity in Philosophical Studies.

Received September 6, 2004; accepted October 6, 2004. 